\title{
On Sylow intersections
}

\section{Ariel Ish-Shalom}

Let $G$ be a finite group, $p$ a prime divisor of $|G|$, and $T$ a p-subgroup of $G$. Define $\sigma(T)$ to be the number of sylow p-subgroups of $G$ containing $T$. Call $T$ a central p-Sylow intersection if for some $\Sigma \subseteq \operatorname{Syl}_{p}(G), T=n\{S \mid S \in \Sigma\}$, and if, in addition, $T$ contains the center of a sylow $p$-subgroup of $G$. This work is inspired and motivated by work of $G$. Stroth [J. Algebra 37 (1975), 1ll-120]. Generalizing an argument of his we describe finite groups in which every central p-Sylow intersection $T$ with $p-\operatorname{rank}(T)>2$ satisfies $\sigma(T) \leq p$.

Related methods yield the description of finite groups in which every central p-Sylow intersection $T$ with $p$-rank $(T) \geq 2$ satisfies $\sigma(T) \leq 2 p$.

\section{Introduction}

Let $G$ be a finite group, $p$ a prime divisor of the order of $G$, and $T$ a $p$-subgroup of $G$. Define $\sigma(T)$ to be the number of sylow p-subgroups of $G$ containing $T$, and $p$-rank $(T)$ to be the maximal number $n$ such that $T$ contains an elementary abelian subgroup of order $p^{n}$. We call $T$ a p-Sylow intersection if for some $\sum \subseteq \operatorname{Syl}_{p}(G)$, $T=n\{S \mid S \in \Sigma\}$, and we call $T$ a central p-Sylow intersection if, in addition, $T$ contains the center of a sylow p-subgroup of $G$.

In a previous paper [9] we proved

THEOREM 1. Let every central p-Sylow intersection $T$ satisfy

Received 12 November 1976. Communicated by Marcel Herzog. This paper is part of the author's PhD thesis done at the University of Tel-Aviv under the supervision of Professor Marcel Herzog. 
$\sigma(T) \leq 2 p$. Then there exists a non identity abelian subgroup, strongly closed in a sylow p-subgroup of $G$ with respect to $G$.

In that paper, [9], we also characterized those groups $G$ in which $\sigma(T) \leq 6$ for every central 2-Sylow intersection $T$.

This work is inspired and motivated by Stroth [10]. In that paper, Stroth gives a detailed characterization of finite groups in which every intersection of two distinct Sylow 2-subgroups is of 2-rank $\leq 2$, and in which there exists such an intersection of 2-rank $=2$. Generalizing an argument of his we prove:

THEOREM 2. Let every central p-Syzow intersection $T$ with $p-\operatorname{rank}(T)>2$ satisfy $\sigma(T) \leq p$. Let $S$ be a sylow p-subgroup of $G$. Then either

(i) $\Omega(Z(S))$ is strongly closed in $S$ with respect to $G$, or

(ii) $p-\operatorname{rank}(S)=2$, or

(iii) there exists some $x \in S$ with $C_{S}(x)$ elementary abelian of order $p^{2}$.

We remark that by Lemma 6 below, the condition of Theorem 2 forces every central $p$-Sylow intersection $T$ with $p$-rank $(T)>2$ to belong to ${ }^{\mathrm{Syl}}{ }_{p}(G)$. We also remark that $p$-groups satisfying conclusion (iii) are discussed in [7], Kapitel III, \$14. For $p=2$, a subgroup $S$ satisfying (iii) is dihedral or semidihedral by Lemma 4 of [11]. Thus we get

COROLLARY 3. Let every central 2-Syzow intersection $T \notin \operatorname{Syl}_{2}(G)$ satisfy 2-rank $(T) \leq 2$. Let $S$ be a Syzow 2-subgroup of $G$. Then either

(i) $\Omega(Z(S))$ is strongly closed in $S$ with respect to $G$, or (ii) $2-\operatorname{rank}(S)=2$.

We remark that for finite simple groups the conclusions of Corollary 3 are in fact equivalent to its assumptions. These finite simple groups were already listed in [10], namely: $L_{2}(q), U_{3}(q), S z(q), q$ even, $L_{2}(q)$, $L_{3}(q), U_{3}(q), q$ odd, $A_{7}, M_{11}$, and simple groups of Janko-Ree type.

In this paper we also prove a generalization of Theorem 1 . 
THEOREM 4. Let every central p-Sylow intersection $T$ with $p-\operatorname{rank}(T) \geq 2$ satisfy $\sigma(T) \leq 2 p$. Let $S$ be a Sylow p-subgroup of $G$. Then either

(i) there exists a non identity abelian subgroup, strongly closed in $S$ with respect to $G$, or

(ii) there exists some $x \in S$ with $C_{S}(x)$ elementary abelian of order $p^{2}$.

Again, for $p=2$, we get

COROLLARY 5. Let every central 2-Sylow intersection $T$ with $2-\operatorname{rank}(T) \geq 2$ satisfy $\sigma(T) \leq 4$. Let $S$ be a Sylow 2-subgroup of $G$. Then either

(i) there exists a non identity abelian subgroup, strongly closed in $S$ with respect to $G$, or

(ii) $S$ is dihedral or semidihedral.

Those finite simple groups satisfying the hypothesis of Corollary 5 are: $L_{2}(q), U_{3}(q), S z(q), q$ even, $L_{2}(q), q \equiv 3,5(\bmod 8)$, and simple groups of Janko-Ree type, as can be verified by [4] and by Remark 8 of $[9]$.

\section{Preliminary results}

LEMMA 6. (i) If $T$ is a p-subgroup of $G$ then $\sigma(T) \equiv 1(\bmod p)$.

(ii) Let $T$ and $T^{\prime}$ be p-Sylow intersections in $G$. If $T \subseteq T^{\prime}$, then $\sigma(T) \geq \sigma\left(T^{\prime}\right)$, and if $T \subset T^{\prime}$, then $\sigma(T)>\sigma\left(T^{\prime}\right)$.

(iii) If $T$ is a p-Sylow intersection satisfying $\sigma(T)=1$, then $T \in \operatorname{Syl}_{p}(G)$.

(iv) If $T$ is a p-Sylow intersection satisfying $\sigma(T)=1+p$, then $N_{S}(T) / T$ is cyclic of order $p$, for every sylow p-subgroup $S$ of $G$ containing $T$.

Proof. Assertion ( $i$ ) is Lemma 6 of [8]. Assertion (ii) is trivial once we notice that any $p$-Sylow intersection $T$ is the intersection of those sylow p-subgroups containing it. Assertion (iii) is also trivial. 
Denote by $\Omega$ the set of $I+p$ Sylow $p$-subgroups of $G$ containing $T$, and take any $S \in \Omega$. The subgroup $N_{S}(T)$ acts by conjugation on $\Omega^{\prime} \equiv \Omega \backslash\{S\}$. If $g \in N_{S}(T)$ stabilizes some $R \in \Omega^{\prime}$, then being a p-element, $g \in N_{S}(T) \cap R \subseteq S \cap R$. But $S \supset S \cap R \supseteq T$ forces $S \cap R=T$ by (i), (ii), and (iii), so that $N_{S}(T) / T$ acts faithfully on $\Omega^{\prime}$. In fact, every $g \in\left(N_{S}(T) / T\right)^{\#}$ acts fixed point freely on $\Omega^{\prime}$, whence $|\Omega|=p$ yields assertion (iv).

The following result is due to Alperin. The first part is Theorem 5.2 of [1], and the second is a strengthening of the corollary in [2], achieved by self suggestive changes in its proof.

THEOREM 7 (Alperin). Let $x$ and $y$ be elements of $S \in \operatorname{Syl}_{p}(G)$, such that $x$ is conjugate to $y$ in $G$. Then there exist central p-Sylow intersections $H_{i} \subseteq S, i=1, \ldots, n$, and elements $t_{i} \in N_{G}\left(H_{i}\right), i=1, \ldots, n$, such that $N_{S}\left(H_{i}\right) \in \operatorname{Syl}_{p}\left(N_{G}\left(H_{i}\right)\right)$, $i=1, \ldots, n$, that $t_{i}$ is a p-element if $H_{i} \subset S$, and that, setting $x_{1}=x, x_{2}=x^{t_{1}}, \ldots, x_{n+1}=x^{t_{1} t_{2} \cdots t_{n}}$, we get $x_{i} \in H_{i}$, $i=1, \ldots, n$, and $x_{n+1}=y$.

Moreover, if $C_{S}(y) \in \operatorname{Syl}_{p}\left(C_{G}(y)\right)$, then we can assure in addition that

(i) $c_{S}\left(x_{i}\right) \subseteq H_{i}, \quad i=1, \ldots, n$, and that

(ii) $\left|C_{S}\left(x_{1}\right)\right| \leq\left|C_{S}\left(x_{2}\right)\right| \leq \ldots \leq\left|c_{S}\left(x_{n+1}\right)\right|$.

Let $S$ be a Sylow p-subgroup of $G$. Denote by $J$ the set of elements in $S \backslash Z(S)$, which are conjugate in $G$ to an element of $\Omega(Z(S))$. Denote by $J^{*}$ the set of those elements $j \in J$ which are conjugate to an element of $\Omega(z(S))$ in ${ }_{G}\left(C_{S}(j)\right)$.

LEMMA 8. (i) $J=\emptyset$ if and only if $\Omega(Z(S))$ is strongly closed in $S$ with respect to $G$.

(ii) For every $j \in J$ there exists some $S^{\prime} \in \operatorname{Syl}_{p}\left(C_{G}(j)\right) \subseteq \operatorname{Syl}_{p}(G)$, 
such that $C_{S}(j)=S \cap S^{\prime}$ (whence $\left.\sigma\left(C_{S}(j)\right)>p\right)$.

(iii) If $J \neq \emptyset$ there exist $j \in J$ and $g, a$ p-element of ${ }^{N}{ }_{G}\left(C_{S}(j)\right)$, such that:

(I) $j \in \Omega\left(z\left(s^{g}\right)\right)$;

(2) $C_{S}(j)=S \cap S^{g}$; and

(3) $N_{S}\left(C_{S}(j)\right) \in \operatorname{Syl}_{p}\left(N_{G}\left(C_{S}(j)\right)\right)$;

in particular, $J \neq \emptyset$ implies $J^{*} \neq \emptyset$.

Proof. Assertion ( $i$ ) is obvious. To satisfy assertion (ii), any $S^{\prime} \in \operatorname{Syl}_{p}\left(C_{G}(j)\right)$ containing $C_{S}(j)$ will do. To prove $(i i i)$ choose some $x \in J$, and some $y \in \Omega(Z(S))$ such that $x$ is conjugate in $G$ to $y$. Now quote Theorem 7 . As $\left|C_{S}(y)\right|>\left|C_{S}(x)\right|$, the set

$\left\{k|| c_{S}\left(x_{k}\right)|<| c_{S}\left(x_{k+1}\right) \mid\right\}$ is not empty; let $i_{0}$ be its maximal element, and set $j=x_{i_{0}}, g=t_{i_{0}}^{-1}$, and $H \equiv H_{i_{0}}$. Clearly $j \in J$, $j^{g^{-1}} \in \Omega(z(S))$, and $C_{S}(j) \subseteq H$. Now $j$ is conjugate in $N_{G}(H)$ to $j^{g^{-1}}$ which is an element of $H \cap Z(S) \subseteq Z(H)$. Thus $H \subseteq C_{S}(j)$ and we are done.

COROLLARY 9. Let $S$ be a Syzow p-subgroup of $G$. If $\sigma(T) \leq p$ for every central p-Sylow intersection $T$ with $p$-rank $(T) \geq 2$, then $\Omega(z(S))$ is strongly closed in $S$ with respect to $G$.

Proof. If $j \in J$, then $T \equiv C_{S}(j)$ is a central p-Sylow intersection with $p-\operatorname{rank}(T) \geq 2$ and $\sigma(T)>p$ by $(i i)$ of Lemma 8 . Thus $J=\varnothing$ and we are through by (i) of Lemma 8 .

We remark that the results of Herzog and Shult [6] and those of Gomi [5] follow from Corollary 9 and Goldschmidt [4].

\section{Proof of Theorem 2}

By Lemma 8 (i) and ( $i i i)$, either conclusion (i) of our theorem holds, or 
or $J^{*} \neq \varnothing$. Thus we may assume the existence of $i \in \Omega(z(S))$ and $j \in J$ such that $i$ is conjugate to $j$ in $N_{G}\left(C_{S}(j)\right)$. By Lerma 8 (ii), $\sigma\left(C_{S}(j)\right)>p$; hence, $C_{S}(j)$ being a central p-Sylow intersection, $p-\operatorname{rank}\left(c_{S}(j)\right)=2$ by the assumption of the theorem. As $z\left(c_{S}(j)\right)$ contains the subgroup $\langle i, j\rangle$, which is elementary abelian of order $p^{2}$, it follows that

$$
\Omega\left(c_{S}(j)\right)=\langle i, j\rangle,
$$

and that $\Omega(z(S))=\langle i\rangle$.

As $C_{S}(\langle i, j\rangle)=C_{S}(j)$, and $N_{S}(\langle i, j\rangle) \supseteq N_{S}\left(C_{S}(j)\right)$, we have that $n_{S}\left(C_{S}(j)\right) / C_{S}(j)$ is a subgroup of $N_{S}(\langle i, j\rangle) / C_{S}((i, j\rangle)$, which is isomorphic to a subgroup of $\mathrm{GL}(2, p)$ - the automorphism group of an elementary abelian group of order $p^{2}$. Thus, being a non-trivial p-group, $N_{S}\left(C_{S}(j)\right) / C_{S}(j)$ is cyclic of order $p$.

Now $N_{G}\left(C_{S}(j)\right)$ acts by conjugation on $\Omega$, the set of all cyclic subgroups of $\langle i, j\rangle$, and we claim that this action is transitive. Indeed, take any $g \in N_{S}\left(C_{S}(j)\right) \backslash C_{S}(j)$. As $g$ is a p-element which does not centralize any element of $\langle i, j\rangle i\rangle$, it follows that $g$ acts fixed point freely on $\Omega \backslash\{\langle i\rangle\}$. Hence $|\Omega|=p+1$ yields that $g$ acts transitively on $\Omega \backslash\{\langle i\rangle\}$. Concluding the fact that $i$ is conjugate to $j$ in $N_{G}\left(C_{S}(j)\right)$ implies that $\langle i\rangle$ is conjugate to $\langle j\rangle$ in $N_{G}\left(C_{S}(j)\right)$ and our claim is proved.

Proceeding with our proof, let us assume first that $N_{S}\left(C_{S}(j)\right) \subset S$. Take any $g \in N_{S}\left(N_{S}\left(C_{S}(j)\right)\right) N_{S}\left(C_{S}(j)\right)$ to get $C_{S}(j) \neq C_{S}\left(j^{g}\right) \subseteq N_{S}\left(C_{S}(j)\right)$, whence $j^{g} \vDash\langle i, j\rangle$ implies that $\left\langle j^{g}\right\rangle^{\#} \subseteq N_{S}\left(C_{S}(j)\right) \backslash C_{S}(j)$. As $N_{S}\left(C_{S}(j)\right) / C_{S}(j)$ is cyclic of order $p$, no element of $\left(j^{g}\right)^{\#}$ has any p-roots in $c_{S}\left(j^{g}\right)$. Hence, by the preceding paragraph, no element of $(i, j)^{\#}$ has any $p$-roots in $C_{S}(j)$. It follows by $(*)$, that 
$C_{S}(j)=\langle i, j\rangle$, and conclusion $(i i i)$ holds.

Assume then that $N_{S}\left(c_{S}(j)\right)=S$, so that $\left|S: C_{S}(j)\right|=p$. Let $K$ be an elementary abelian subgroup of $S$ of maximal order. If $\left|K \cap C_{S}(j)\right| \geq p^{2}$, then $\left(^{*}\right)$ forces $j \in K \cap C_{S}(j)$, whence $K \subseteq C_{S}(j)$, and (*) yields $|K| \leq p^{2}$. If $\left|K \cap C_{S}(j)\right| \leq p$, then $\left|K: K \cap C_{S}(j)\right| \leq p$ implies that again $|K| \leq p^{2}$. Anyhow, conclusion (ii) holds and we are done.

\section{Proof of Theorem 4}

If $p-\operatorname{rank}(Z(S))>1$, then by our assumption $\sigma(z(S)) \leq 2 p$, whence conclusion $(i)$ holds by Theorem 1 . Hence we may assume that $p-\operatorname{rank}(z(S))=1$, whence $\Omega(z(S))$ is cyclic of order $p$, say, $\Omega(z(S))=\langle i\rangle$.

If $j \in J$, then $p-\operatorname{rank}\left(C_{S}(j)\right) \geq 2$. Thus, being a central p-Sylow intersection, $p<\sigma\left(C_{S}(j)\right) \leq 2 p$. Hence Lemma 6 (i) yields that $\sigma\left(C_{S}(j)\right)=1+p$, and Lemma 8 (iii) that $N_{S}\left(C_{S}(j)\right) / C_{S}(j)$ is cyclic of order $p$.

We claim that

(*) if $j_{1} \in J^{*}, j_{2} \in J$, and $\left|c_{S}\left(j_{1}\right): C_{S}\left(j_{1}\right) \cap C_{S}\left(j_{2}\right)\right|=p$, then (ii) holds.

Indeed, $\quad c_{S}\left(j_{1}\right) \cap C_{S}\left(j_{2}\right)$ is a central $p$-Sylow intersection strictly contained in $c_{S}\left(j_{1}\right)$. Thus $1+p=\sigma\left(c_{S}(j)\right)<\sigma\left(c_{S}\left(j_{1}\right) \cap c_{S}\left(j_{2}\right)\right)$ by Lemma $6(i i)$. Hence, by Lemma $6(i), 2 p<\sigma\left(C_{S}\left(j_{1}\right) \cap C_{S}\left(j_{2}\right)\right)$, so that $p-\operatorname{rank}\left(C_{S}\left(j_{1}\right) \cap C_{S}\left(j_{2}\right)\right)=1$, and $\Omega\left(C_{S}\left(j_{1}\right) \cap C_{S}\left(j_{2}\right)\right)=\Omega(z(S))$. Now $\left\langle j_{1}\right)^{\#} \cap \Omega(z(S))=\varnothing$, so that by the assumption of $\left(^{*}\right)$, no element of $\left\langle j_{1}\right\rangle^{\#}$ has any p-roots in $C_{S}\left(j_{1}\right)$. As $j_{1} \in J^{*}$, so is the case with the elements of $\Omega(z(S))^{\#}$. Thus, the fact that $\Omega\left(C_{S}\left(j_{1}\right) \cap C_{S}\left(j_{2}\right)\right)=\Omega(z(S))$ 
implies that $C_{S}\left(j_{1}\right) \cap C_{S}\left(j_{2}\right)=\Omega(z(S))=\langle i\rangle$, so that $C_{S}\left(j_{1}\right)$ is elementary abelian of order $p^{2}$, and claim $(*)$ is proved.

By Lemma $8(i)$ and $(i i i)$, either conclusion $(i)$ of our theorem holds, or $J^{*} \neq \varnothing$. Thus we may assume the existence of some $j_{0} \in J^{*}$. If $c_{S}\left(j_{0}\right)$ is not normal in $s$, take $g \in N_{S}\left(N_{S}\left(c_{S}\left(j_{0}\right)\right)\right) \backslash N_{S}\left(c_{S}\left(j_{0}\right)\right)$. Then . $j_{0}^{g} \in J$, and $c_{S}\left(j_{0}\right) \neq c_{S}\left(j_{0}^{g}\right) \subseteq N_{S}\left(c_{S}\left(j_{0}\right)\right)$. As $N_{S}\left(c_{S}\left(j_{0}\right)\right) / c_{S}\left(j_{0}\right)$ is cyclic of order $p$, it follows that $\left|c_{S}\left(j_{0}\right): c_{S}\left(j_{0}\right) \cap c_{S}\left(j_{0}^{g}\right)\right|=p$, and (ii) holds by (*). Hence we may assume that $c_{S}\left(j_{0}\right)$ is normal in $S$, so that $\left|S: C_{S}\left(j_{0}\right)\right|=p$. By the same argument we may assume now that $C_{S}\left(j_{0}\right)$ is normal in $N_{G}(S)$, for otherwise we take any $g \in N_{G}(S) \backslash N_{G}\left(C_{S}\left(j_{0}\right)\right)$, and repeat the process, to show that (ii) holds. We may also assume that $J^{*} \subseteq C_{S}\left(j_{0}\right)$. If this is not the case, take in (*) any $j \in J * \backslash C_{S}\left(j_{0}\right)$ as $j_{1}$, and $j_{0}$ as $j_{2}$, and conclude that (ii) holds.

We claim now, that $\Omega\left(z\left(c_{S}\left(j_{0}\right)\right)\right)$ is strongly closed in $S$ with respect to $G$. To prove it, suppose that there exist $j^{\prime} \in S \backslash \Omega\left(z\left(C_{S}\left(j_{0}\right)\right)\right)$, and $k^{\prime} \in \Omega\left(z\left(C_{S}\left(j_{0}\right)\right)\right)$, such that $j^{\prime}$ is conjugate to $k^{\prime}$ in $G$. By Theorem 7 we may assume that there exists a central p-Sylow intersection $H$ and elements $j \in H \backslash \Omega\left(z\left(c_{S}\left(j_{0}\right)\right)\right)$ and $k \in H \cap \Omega\left(z\left(C_{S}\left(j_{0}\right)\right)\right)$, such that $j$ is conjugate to $k$ in $N_{G}(H)$.

Assume first that $k \vDash \Omega(z(S))$. Then $\langle k, \Omega(z(S))\rangle \subseteq H \cap c_{S}\left(j_{0}\right) \subseteq H$ yields that $1 \leq \sigma(H) \leq \sigma\left(H \cap C_{S}\left(j_{0}\right)\right) \leq 2 p$ by the assumption of the theorem. Thus, by Iremme 6 , either $\sigma(H)=1$, whence $H=S$, or $\sigma(H)=\sigma\left(H \cap C_{S}\left(j_{0}\right)\right)=\sigma\left(C_{S}\left(j_{0}\right)\right)$, and $H=C_{S}\left(j_{0}\right)$. But $\Omega\left(z\left(C_{S}\left(j_{0}\right)\right)\right)$ is normal in $\left\langle N_{G}(S), N_{G}\left(C_{S}\left(j_{0}\right)\right)\right\rangle$, a contradiction.

Thus we may assume that $k \in \Omega(Z(S))$ so that $j \in J$. Moreover, $\{j, \Omega(z(S))\rangle \subseteq H \cap C_{S}(j) \subseteq H$ yields as before that either $H=S$ or 
$H=C_{S}(j)$. The first case is impossible as $j k \Omega(z(S))$ and $k \in \Omega(z(S))$.

Thus $j \in J^{*}$, and as $J^{*} \subseteq C_{S}\left(j_{0}\right)$, we have

$$
\left\langle j, \Omega\left(z\left(c_{S}\left(j_{0}\right)\right)\right)\right\rangle \subseteq c_{S}(j) \cap c_{S}\left(j_{0}\right) \subseteq c_{S}(j) .
$$

Using again the above argument yields $C_{S}(j)=C_{S}\left(j_{0}\right)$, so that $j \in \Omega\left(z\left(C_{S}\left(j_{0}\right)\right)\right)$, a contradiction. Thus our claim is proved and conclusion (i) of our theorem holds.

We remark that the condition $\sigma(T) \leq 2 p$ in the assumption of the theorem is needed only to assure that if $T \subset T^{\prime \prime} \subseteq S \in \operatorname{Syl}{ }_{p}(G)$, where $T^{\prime}$ is a p-Sylow intersection, then

(i) $N_{S}(T) / T$ is cyclic of order $p$, and

(ii) $T^{\prime}=S$ (see Lemma 6$)$.

\section{References}

[1] J.L. Alperin, "Sylow intersections and fusion", J. Algebra 6 (1967), 222-241.

[2] J.L. Alperin, "Up and down fusion", J. Algebra 28 (1974), 206-209.

[3] J.L. Alperin, Richard Brauer, Daniel Gorenstein, "Finite simple groups of 2-rank two", Scripta Math. 29 (1973), 191-214.

[4] David M. Goldschmidt, "2-fusion in finite groups", Ann. of Math. (2) 99 (1974), 70-117.

[5] Kensaku Gomi, "Finite groups with central Sylow 2-intersections", $J$. Math. Soc. Japan 25 (1973), 342-355.

[6] Marcel Herzog and Ernest Shult, "Groups with central 2-Sylow intersections of rank at most one", Proc. Amer. Math. Soc. 38 (1973), 465-470.

[7] B. Huppert, Endliche Gruppen I (Die Grundlehren der mathematischen Wissenschaften, 134. Springer-Verlag, Berlin, Heidelberg, New York, 1967). 
[8] Ariel Ish-Shalom, "On 2-Sylow intersections", Israel J. Math. 18 $(1974), 235-242$.

[9] Ariel Ish-Shalom, "On central 2-Sylow intersections", submitted.

[10] G. Stroth, "Eine Kennzeichnung der 2I-Gruppen", J. AZgebra 37 (1975), 111-120.

[11] Michio Suzuki, "A characterization of simple groups $L F(2, p)$ ", $J$. Fac. Sci. Univ. Tokyo Sect. I 6 (1951), 259-293.

Department of Mathematics,

Tel-Aviv University,

Tel-Aviv,

|srael. 